\title{
What microscopy can tell us about Alzheimer's and related diseases.
}

\author{
M E Welland
}

Nanoscience Centre, University of Cambridge, 11 J J Thomson Ave., Cambridge CB3 OFF, UK.

The atomic force microscope can uniquely determine the mechanical properties of surfaces and structures with nanometer spatial resolution. This combination of imaging and mechanical characterization has been used to effect an unprecedented insight into the formation of the nanostructures found deposited in the human body in diseases such as Alzheimer's and Parkinson's. In turn this provides for an understanding of some of the challenges in both diagnostics and therapies in respect of disease management.

In the so-called Amyloid diseases, including Alzheimer's and Parkinson's, a ubiquitous characteristic of the diseased state is the deposition of plaque in the relevant body organ. The plaque consists of nanowires of protein, fibrils, a few nanometers in diameter and up to microns in length. Fibrils form when natural proteins misfold away from their native state and take up a configuration that allows for sequential sequestration of protein monomer into sheets of protein that stack to form fibrils. As such fibrils are of a scale where the tools of nanoscience, specifically scanned probe microscopy, are particularly efficacious as they combine sub-nanometre resolution operation in vitro. Of specific interest is the ability of probe microscopy to measure the mechanical properties of fibrils since both structural integrity and factors such as fracture toughness depend directly upon structure. They are also properties that are entirely relevant in the pathogenesis and treatment of the disease itself.

Our technique is based on growing fibrils from the target protein, a-beta in the case of Alzheimer's, in-vitro by essentially controlling the chemistry and $\mathrm{pH}$ of the solution. [1] Fibrils nucleate and attach to the surface that is then imaged in-situ with an atomic force microscope. Structural perfection of individual fibrils is determined by observing the helical coherence along the fibril length; a structural defect would cause a phase shift in the helical pitch. Figure 1. Averaging over many fibrils allows for a protein specific correlation length to be derived. Remarkably, this length indicates a very high degree of $1 \mathrm{D}$ perfection along the fibril length for a range of proteins with a defect density comparable to the best protein crystals. [2,3] This degree of structural integrity has important consequences on mechanical stability. To explore this we deposited fibrils on a surface which had been previously patterned with grooves approximately $90 \mathrm{~nm}$ in width. Where a fibril lay across such grooves we were able to bend the fibril by pressing the tip of the atomic force microscope in a controllable fashion; identical to a three-point beam test. From the deflection of the fibril as a function of load and position we are able to determine the bulk and shear modulii. Additionally, by increasing the force on the fibril we can effect fracture thereby determining the fracture toughness of fibrils. Table 1.

We have further advanced these types of measurement by a combination of using statistical fluctuations in fibril shape and dynamic light scattering so that we have a comprehensive and quantitative understanding of fibril mechanics. [4,5] In turn these measurements demonstrate the extraordinary strength of fibrils, not dissimilar to spider's silk, that provide a basis for understanding their stability against therapies to dissolve them. The fracture properties show that in understanding the kinetics of plaque accretion the fracture rate is a dominant factor. 


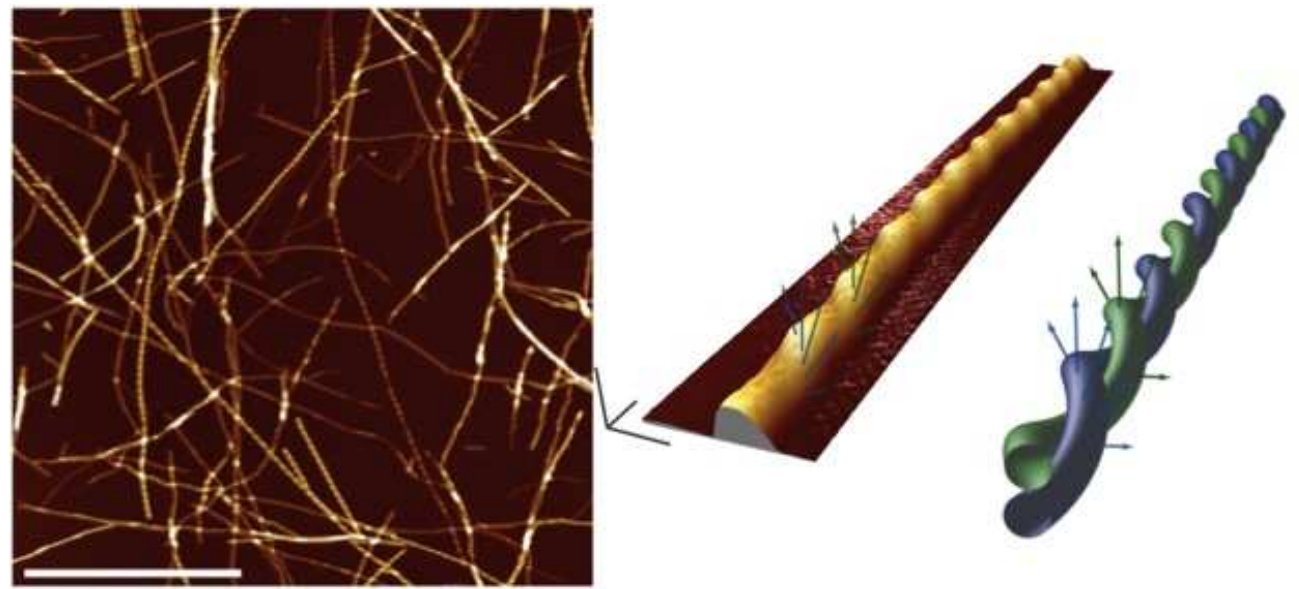

Figure 1. Left, AFM image of insulin fibrils, scale bar $1 \mu \mathrm{m}$. Fibril diameter $3.1 \mathrm{~nm}$ (single filament) and 6.2nm (double filament). Right, AFM image of double filament fibril with cartoon of helical structure indicating surface normals used in determining correlation of helicity.

\begin{tabular}{|c|c|c|c|c|c|}
\hline Property & Force spectroscopy & Image analysis & Rubber(22) & $\operatorname{Silk}(21)$ & Steel(22) \\
\hline Ultimate strength $(\mathrm{GPa})$ & $0.6 \pm 0.4$ & & $0.02-0.04$ & $1-1.5$ & $0.6-1.8$ \\
\hline Compensated strength $(\mathrm{GPa} \mathrm{cm} / \mathrm{g})$ & $0.4 \pm 0.3$ & & $0.002-0.004$ & $0.8-1.2$ & $0.1-0.2$ \\
\hline Young's modulus (GPa) & $3.3 \pm 0.4$ & $6.3 \pm 4.5$ & $10^{-3}-10^{-2}$ & $1-10$ & 200 \\
\hline Persistence length $(\mu \mathrm{m})$ & $22 \pm 3$ & $42 \pm 30$ & & & \\
\hline Bending rigidity $\left(\mathrm{N} \mathrm{m}^{2}\right)$ & $9.1 \pm 1 \cdot 10^{-26}$ & $1.7 \pm 1.2 \cdot 10^{-25}$ & & & \\
\hline Shear modulus (GPa) & $0.28 \pm 0.2$ & $0.13 \pm 0.01(8)$ & & & \\
\hline Torsional rigidity $\left(\mathrm{N} \mathrm{m}^{2}\right)$ & $1.6 \pm 1.1 \cdot 10^{-26}$ & $0.8 \pm 0.06 \cdot 10^{-26}(8)$ & & & \\
\hline
\end{tabular}

Table 1. Mechanical properties of insulin fibrils determined by atomic force microscopy.

References.

[1]. J. F. Smith, et al, PNAS, USA 11315806 (2006)

[2]. T. P. J. Knowles, et al, PRL, 96, 238301 (2006)

[3]. T. P. J. Knowles, et al, Nanotechnology 18044031 (2007)

[4]. T. P. J. Knowles, et al, PNAS, 10410016 (2007)

[5]. T. P. J. Knowles, et al, Science, 3181900 (2007) 